\title{
Parasitology: United Kingdom National Quality Assessment Scheme
}

\author{
M Hawthorne, P L Chiodini, J J S Snell, A H Moody, A Ramsay
}

\begin{abstract}
Aims: To assess the results from parasitology laboratories taking part in a quality assessment scheme between 1986 and 1991; and to compare performance with repeat specimens.

Methods: Quality assessment of blood parasitology, including tissue parasites ( $=444 ; 358$ UK, 86 overseas), and faecal parasitology, including extra-intestinal parasites (n $=205$; $141 \mathrm{UK}, 64$ overseas), was performed.

Results: Overall, the standard of performance was poor. A questionnaire distributed to participants showed that a wide range of methods was used, some of which were considered inadequate to achieve reliable results. Teaching material was distributed to participants from time to time in an attempt to improve standards. Conclusions: Since the closure of the IMLS fellowship course in 1972, fewer opportunities for specialised training in parasitology are available: more training is needed. Poor performance in the detection of malarial parasites is mainly attributable to incorrect speciation, misidentification, and lack of equipment such as an eyepiece graticule.
\end{abstract}

(F Clin Pathol 1992;45:968-974)

The United Kingdom National External Quality Assessment Scheme for Microbiology (NEQAS), which covers many areas of microbiology, has already been described. ${ }^{12}$ As part of this scheme, simulated specimens are despatched to participants, who examine them and report their results to the Quality Assurance Laboratory (QAL). Before 1986 specimens containing parasites were occasionally distributed as part of the general bacteriology quality assessment scheme, there being no separate subscheme for parasitology. Several factors, including retirement of pathology staff who had gained considerable overseas experience in former years, allied to diminished overseas experience of trainee pathologists and closure of the IMLS fellowship course in parasitology in 1972, has led to the gradual decline in the standard of diagnostic parasitology in the United Kingdom. This trend was noted through results from earlier NEQAS distributions, discussions with laboratory staff around the country, an increase in misdiagnoses and more routine work being referred to the Hospital for Tropical Diseases, London
(HTD). The parasitology of the immunocompromised is also becoming increasingly important and not all laboratories may be prepared to tackle rare opportunistic infections.

To help remedy these deficiencies, in 1986 QAL (then part of the (Division of Microbiological Reagents and Quality DMRQC) together with the Department of Clinical Parasitology, HTD, began to develop a quality assessment control) scheme for parasitology. Two subschemes were established: blood parasitology, including tissue parasites and faecal parasitology, including extra-intestinal parasites. Objectives of the parasitology schemes were to provide participants with a variety of specimen material to increase awareness and skills in this specialty, and to educate participants through the production of teaching sheets describing unfamiliar parasites and containing recommendations for standardised methods.

To facilitate access to specimen material and specialised expertise, the day to day functions of the scheme were located at HTD with administrative support based at QAL. Distributions began on a regular basis in January 1987. Since June 1991, NEQAS parasitology has also regularly supplied blood films for distribution by the NEQAS haematology scheme to haematology departments.

\section{Methods}

PARTICIPANTS

A total of 444 laboratories participate in the faecal parasitology scheme of which 358 are in the United Kingdom and 86 are overseas. In the blood parasitology scheme 205 laboratories participate, 141 from the United Kingdom and 64 from overseas.

The parasites distributed are shown in tables 1 to 4. Most material distributed was obtained from patients with known infections and was selected on the basis of the presence of adequate numbers of parasites, with characteristic morphological features and sufficient quantities of specimen. Where naturally infected material was not available, alternative sources were used. Trichomonas vaginalis trophozoites were cultured in TYl-S-33 medium $^{3}$ from which smears were prepared, dried, fixed in methanol and distributed unstained. Giardia lamblia trophozoites were cultured similarly, fixed in formalin, and dispensed into phials before distribution as a simulated jejunal fluid. Filariform larvae of Hookworm spp were obtained by the Harada-Mori filter paper strip culture $^{45}$ and Strongyloides stercoralis by char-
Correspondence to:

P L Chiodini

Accepted for publication 6 April 1992 
Table 1 Single blood and tissue parasites distributed and participants' results

\begin{tabular}{|c|c|c|c|c|c|c|c|c|}
\hline $\begin{array}{l}\text { Specimen } \\
\text { number }\end{array}$ & Species & $\begin{array}{l}\text { Number of } \\
\text { laboratories }\end{array}$ & $\begin{array}{l}\text { Correct genus } \\
\text { species, and stage }\end{array}$ & $\begin{array}{l}\text { Correct genus } \\
\text { only }\end{array}$ & $\begin{array}{l}\text { Incorrect } \\
\text { species } \\
\text { or stage }\end{array}$ & $\begin{array}{l}\text { Unexpected } \\
\text { parasite only }\end{array}$ & None found & $\begin{array}{l}\text { Multiple parasites, } \\
\text { genera, species } \\
\text { or stages }\end{array}$ \\
\hline 1336 & Plasmodium vivax & 80 & $\begin{array}{l}44 \\
(55 \cdot 0 \%)\end{array}$ & $\stackrel{9}{(11 \cdot 2 \%)}$ & $\begin{array}{l}15 \\
(18 \cdot 8 \%)\end{array}$ & & & 12 \\
\hline 1686 & Plasmodium vivax & 116 & $\begin{array}{l}91 \\
(78 \cdot 4 \%)\end{array}$ & $\begin{array}{l}7 \\
(6 \cdot 0 \%)\end{array}$ & $\begin{array}{l}9 \\
(7 \cdot 8 \%)\end{array}$ & & & $\begin{array}{l}9 \\
(7 \cdot 8 \%)\end{array}$ \\
\hline 1917 & Plasmodium vivax & 119 & $\begin{array}{l}93 \\
(78 \cdot 2 \%)\end{array}$ & $\begin{array}{l}3 \\
(2 \cdot 5 \%)\end{array}$ & $\begin{array}{c}5 \\
(4 \cdot 2 \%)\end{array}$ & $\stackrel{1}{(0 \cdot 8 \%)}$ & & $\begin{array}{l}17 \\
(14 \cdot 3 \%)\end{array}$ \\
\hline 2256 & Plasmodium vivax & 138 & $\begin{array}{l}102 \\
(73.9 \%)\end{array}$ & $\begin{array}{l}5 \\
(3 \cdot 6 \%)\end{array}$ & $(12 \cdot 3 \%)$ & & $\stackrel{2}{(1 \cdot 5 \%)}$ & $\begin{array}{l}12 \\
(8 \cdot 7 \%)\end{array}$ \\
\hline 1435 & Plasmodium malariae & 83 & $\begin{array}{l}55 \\
(66 \cdot 3 \%)\end{array}$ & $\begin{array}{l}10 \\
(12 \cdot 0 \%)\end{array}$ & $\begin{array}{l}14 \\
(16.9 \%)\end{array}$ & & $\begin{array}{l}3 \\
(3 \cdot 6 \%)\end{array}$ & $(1 \cdot 2 \%)$ \\
\hline 1957 & Plasmodium malariae & 113 & $\begin{array}{l}81 \\
(71 \cdot 7 \%)\end{array}$ & $\begin{array}{l}8 \\
(7 \cdot 0 \%)\end{array}$ & $\begin{array}{l}13 \\
(11 \cdot 5 \%)\end{array}$ & $\begin{array}{l}7 \\
(6 \cdot 2 \%)\end{array}$ & $\begin{array}{c}3 \\
(2 \cdot 7 \%)\end{array}$ & $\begin{array}{l}1 \\
(0.9 \%)\end{array}$ \\
\hline 2178 & Plasmodium malariae & 137 & $\begin{array}{l}99 \\
(72 \cdot 3 \%)\end{array}$ & $\begin{array}{c}8 \\
(5 \cdot 8 \%)\end{array}$ & $\begin{array}{l}18 \\
(13 \cdot 1 \%)\end{array}$ & $\begin{array}{l}1 \\
(0 \cdot 7 \%)\end{array}$ & $\begin{array}{l}9 \\
(6 \cdot 6 \%)\end{array}$ & 2 \\
\hline 1628 & Plasmodium ovale & 111 & $\begin{array}{l}59 \\
(53 \cdot 2 \%)\end{array}$ & 17 & $\begin{array}{l}31 \\
(27 \cdot 9 \%)\end{array}$ & $\begin{array}{l}1 \\
(0.9 \%)\end{array}$ & $(0.9 \%)$ & $\begin{array}{l}2 \\
(1 \cdot 8 \%)\end{array}$ \\
\hline 1384 & Plasmodium falciparum * & 84 & $\begin{array}{l}66 \\
(78 \cdot 6 \%)\end{array}$ & $\begin{array}{l}7 \\
(8 \cdot 3 \%)\end{array}$ & $\begin{array}{l}6 \\
(7 \cdot 1 \%)\end{array}$ & & & $\begin{array}{l}5 \\
(6 \cdot 0 \%)\end{array}$ \\
\hline 2363 & Plasmodium falciparum* & 127 & $\begin{array}{l}90 \\
(70 \cdot 9 \%)\end{array}$ & - & $\stackrel{1}{(0 \cdot 8 \%)}$ & & & $\begin{array}{l}36 \\
(28 \cdot 3 \%)\end{array}$ \\
\hline 1516 & Trypanosoma brucei sp & 103 & $\begin{array}{l}40 \\
(38 \cdot 8 \%)\end{array}$ & $\begin{array}{l}61 \\
(59 \cdot 2 \%)\end{array}$ & $\stackrel{2}{2}$ & & & \\
\hline 1976 & Trypanosoma brucei sp & 125 & $\begin{array}{l}91 \\
(72 \cdot 8 \%)\end{array}$ & $\begin{array}{l}20 \\
(16.0 \%)\end{array}$ & $\begin{array}{l}10 \\
(8 \cdot 0 \%)\end{array}$ & $\begin{array}{l}1 \\
(0 \cdot 8 \%)\end{array}$ & $\underset{(1 \cdot 6 \%)}{2}$ & $\begin{array}{l}1 \\
(0 \cdot 8 \%)\end{array}$ \\
\hline 1977 & Trypanosoma cruzi & 125 & $\begin{array}{l}97 \\
(77 \cdot 6 \%)\end{array}$ & 18 & $\begin{array}{l}8 \\
(6 \cdot 4 \%)\end{array}$ & & & $\begin{array}{l}2 \\
(1 \cdot 6 \%)\end{array}$ \\
\hline 2201 & Leishmania sp & 134 & $\begin{array}{l}124 \\
(92 \cdot 6 \%)\end{array}$ & $\begin{array}{l}1 \\
(0 \cdot 7 \%)\end{array}$ & & $\begin{array}{l}3 \\
(2 \cdot 2 \%)\end{array}$ & $\begin{array}{l}5 \\
(3 \cdot 8 \%)\end{array}$ & $(0 \cdot 7 \%)$ \\
\hline 1575 & Loa loa & 110 & $\begin{array}{l}54 \\
(49 \cdot 1 \%)\end{array}$ & $\begin{array}{l}25 \dagger \\
(22 \cdot 7 \%)\end{array}$ & & $\begin{array}{l}23 \star \star \\
(20.9 \%)\end{array}$ & $\begin{array}{c}8 \\
(7 \cdot 3 \%)\end{array}$ & \\
\hline 1847 & Loa loa & 117 & $\begin{array}{l}97 \\
(82 \cdot 9 \%)\end{array}$ & $8 \dagger$ & & $\begin{array}{l}12 \star \star \\
(10 \cdot 3 \%)\end{array}$ & & \\
\hline 2151 & Loa loa & 133 & $\begin{array}{l}72 \\
(54 \cdot 1 \%)\end{array}$ & $\begin{array}{l}15 t \\
(11 \cdot 3 \%)\end{array}$ & & $\begin{array}{l}36^{\star \star} \\
(27 \cdot 1 \%)\end{array}$ & $\begin{array}{l}10 \\
(7 \cdot 5 \%)\end{array}$ & \\
\hline 2370 & Onchocerca volvulus & 122 & $\begin{array}{l}79 \\
(64 \cdot 8 \%)\end{array}$ & $\begin{array}{l}10 \dagger \\
(8 \cdot 2 \%)\end{array}$ & $\begin{array}{l}3 \\
(2 \cdot 4 \%)\end{array}$ & $\begin{array}{l}24 \star \star \\
(19 \cdot 7 \%)\end{array}$ & & $\stackrel{6}{6}(4 \cdot 9 \%)$ \\
\hline
\end{tabular}

^For identification only, not assessment of parasitaemia.

†The reporting of "Microfilariae present" has been considered equivalent to genus level identification.

Specimen 2285 was a mixed sample containing Plasmodium falciparum and Plasmodium vivax. The following results were obtained:

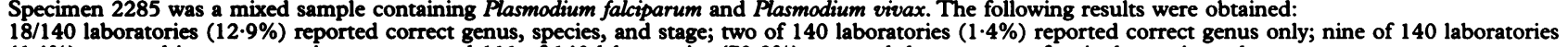

$(6.4 \%)$ reported incorrect species or stage; and 111 of 140 laboratories $(79.3 \%)$ reported the presence of a single species only.

Two negative samples (no parasites present) were distributed and the following results were obtained:

specimen 1763: $93 / 96$ laboratories $(96.9 \%)$ reported no parasites present; three of 93 laboratories $(3 \cdot 1 \%)$ reported an unexpected parasite.

specimen 2344: $134 / 139$ laboratories $(96 \cdot 4 \%)$ reported no parasites present; five of 139 laboratories $(3 \cdot 6 \%)$ reported an unexpected parasite.

Table 2 Percentage parasitaemias of Plasmodium falciparum and participants' results

\begin{tabular}{|c|c|c|c|c|}
\hline $\begin{array}{l}\text { Specimen } \\
\text { number }\end{array}$ & $\begin{array}{l}\text { Actual } \\
\text { \% parasitaemia }\end{array}$ & $\begin{array}{l}\text { Number of laboratories } \\
\text { (total examining) }\end{array}$ & $\begin{array}{l}\text { \% parasitaemia } \\
\text { reported }\end{array}$ & $\begin{array}{l}\text { Number of laboratories } \\
\text { reporting result }\end{array}$ \\
\hline 1956 & $<1 \%$ & 107 & $\begin{array}{l}<2 \% \\
5-10 \%\end{array}$ & $\begin{array}{l}105 \\
(98 \cdot 0 \%) \\
2 \\
(2 \cdot 0 \%)\end{array}$ \\
\hline 1738 & $6.5 \%$ & 109 & $\begin{array}{l}<4 \cdot 5 \% \\
4 \cdot 5-8 \cdot 5 \% \\
8 \cdot 6-10 \cdot 0 \% \\
10 \cdot 1-12 \cdot 0 \% \\
12 \cdot 0-15 \cdot 0 \% \\
15 \cdot 1-20 \cdot 0 \% \\
>20 \%\end{array}$ & $\begin{array}{c}1 \\
(0 \cdot 9 \%) \\
41.6 \%) \\
(37 \cdot 6 \%) \\
37 \\
(33 \cdot 9 \%) \\
7 \\
(6 \cdot 4 \%) \\
16 \\
(14 \cdot 7 \%) \\
4 \\
(3 \cdot 7 \%) \\
3 \\
(2.8 \%)\end{array}$ \\
\hline 2323 & $23 \%$ & 127 & $\begin{array}{r}9-11 \% \\
15-17 \% \\
18-20 \% \\
21-25 \% \\
26-28 \% \\
29-31 \% \\
32-34 \% \\
35-37 \% \\
38-40 \% \\
>40 \%\end{array}$ & $\begin{array}{c}1 \\
(0 \cdot 8 \%) \\
2 \\
(1 \cdot 6 \%) \\
7 \\
(5 \cdot 5 \%) \\
42 \\
(33.1 \%) \\
24 \\
(18 \cdot 9 \%) \\
23 \\
(18 \cdot 1 \%) \\
7 \\
(5 \cdot 5 \%) \\
6 \\
(4 \cdot 7 \%) \\
10 \\
(7.9 \%) \\
5 \\
(3.9 \%)\end{array}$ \\
\hline
\end{tabular}


Table 3 Single faecal parasites distributed and participants' results

\begin{tabular}{|c|c|c|c|c|c|c|c|c|}
\hline $\begin{array}{l}\text { Specimen } \\
\text { number }\end{array}$ & Species & $\begin{array}{l}\text { Number of } \\
\text { laboratories }\end{array}$ & $\begin{array}{l}\text { Correct genus } \\
\text { species, and stage }\end{array}$ & $\begin{array}{l}\text { Correct genus } \\
\text { only }\end{array}$ & $\begin{array}{l}\text { Incorrect species, } \\
\text { stage, or partial } \\
\text { identification }\end{array}$ & $\begin{array}{l}\text { Unexpected } \\
\text { parasite only }\end{array}$ & None found & $\begin{array}{l}\text { Multiple parasites, } \\
\text { genera, species } \\
\text { or stages }\end{array}$ \\
\hline $\begin{array}{l}\text { Nematodes: } \\
1383\end{array}$ & Ascaris lumbricoides & 338 & 258 & & & 9 & 63 & 8 \\
\hline 1599 & Ascaris lumbricoides & 340 & $\begin{array}{l}(76 \cdot 3 \%) \\
314 \\
(92 \cdot 3 \%)\end{array}$ & & & $\begin{array}{l}(2 \cdot 7 \%) \\
5 \\
(1 \cdot 5 \%)\end{array}$ & $\begin{array}{l}(18 \cdot 6 \%) \\
15 \\
(4 \cdot 4 \%)\end{array}$ & $\begin{array}{l}(2 \cdot 4 \%) \\
6 \\
(1 \cdot 8 \%)\end{array}$ \\
\hline 1710 & Ascaris lumbricoides & 343 & $\begin{array}{l}333 \\
(97 \cdot 1 \%)\end{array}$ & & $\begin{array}{l}1 \\
(0.3 \%)\end{array}$ & $(0.3 \%)$ & 2 & $\begin{array}{l}6 \\
(1 \cdot 7 \%)\end{array}$ \\
\hline 1955 & Ascaris lumbricoides & 330 & $\begin{array}{l}308 \\
(93 \cdot 3 \%)\end{array}$ & & & $\begin{array}{l}5 \\
(1.5 \%)\end{array}$ & $\begin{array}{l}15 \\
(4 \cdot 6 \%)\end{array}$ & $(0.6 \%)$ \\
\hline 1626 & Hookworm sp & 341 & $\begin{array}{l}325 \\
(95 \cdot 3 \%)\end{array}$ & & & 6 & $\begin{array}{l}8 \\
(2 \cdot 3 \%)\end{array}$ & 2 \\
\hline 2368 & Hookworm sp & 343 & $\begin{array}{l}285 \\
(83 \cdot 1 \%)\end{array}$ & & $\begin{array}{l}3 \\
(0.9 \%)\end{array}$ & $\begin{array}{l}6 \\
(1 \cdot 7 \%)\end{array}$ & $\begin{array}{l}23 \\
(6 \cdot 7 \%)\end{array}$ & $\begin{array}{l}26 \\
(7 \cdot 6 \%)\end{array}$ \\
\hline 1737 & $\begin{array}{l}\text { Hookworm sp } \\
\text { (filariform larvae) }\end{array}$ & 342 & $\begin{array}{l}180 \\
(52 \cdot 6 \%)\end{array}$ & & $\begin{array}{l}17 \\
(5 \cdot 0 \%)\end{array}$ & $\begin{array}{l}130 \dagger \\
(38.0 \%)\end{array}$ & $(0.3 \%)$ & $(4 \cdot 1 \%)$ \\
\hline 1514 & $\begin{array}{l}\text { Strongyloides stercoralis } \\
\text { (rhabditiform larvae) }\end{array}$ & 327 & $\begin{array}{l}284 \\
(86.9 \%)\end{array}$ & & 14 & $7(2 \cdot 1 \%)$ & 16 & $\begin{array}{l}6 \\
(1 \cdot 8 \%)\end{array}$ \\
\hline 2255 & $\begin{array}{l}\text { Strongyloides stercoralist† } \\
\text { (filariform larvae) }\end{array}$ & 321 & 127 & & $(36 \cdot 1 \%)$ & $(11 \cdot 8 \%)$ & $(12 \cdot 5 \%)$ & \\
\hline 1600 & Enterobius vermicularis & 339 & $\begin{array}{l}299 \\
(88 \cdot 2 \%)\end{array}$ & & & $\begin{array}{l}5 \\
(1 \cdot 5 \%)\end{array}$ & $\begin{array}{l}35 \\
(10.3 \%)\end{array}$ & \\
\hline $\begin{array}{l}\text { Cestodes: } \\
1335\end{array}$ & Hymenolepis nana & 314 & $\begin{array}{l}192 \\
(61 \cdot 1 \%)\end{array}$ & $\begin{array}{l}14 \\
(4 \cdot 5 \%)\end{array}$ & $\begin{array}{l}4 \\
(1 \cdot 3 \%)\end{array}$ & $\begin{array}{l}18 \\
(5 \cdot 7 \%)\end{array}$ & $\begin{array}{l}32 \\
(10 \cdot 2 \%)\end{array}$ & $\begin{array}{l}54 \\
(17 \cdot 2 \%)\end{array}$ \\
\hline 2369 & Hymenolepis nana & 343 & $\begin{array}{l}258 \\
(75 \cdot 3 \%)\end{array}$ & $(2.0 \%)$ & $\begin{array}{l}16 \\
(4 \cdot 7 \%)\end{array}$ & $\begin{array}{l}7 \\
(2.0 \%)\end{array}$ & $\begin{array}{l}24 \\
(7 \cdot 0 \%)\end{array}$ & $\begin{array}{l}31 \\
(9 \cdot 0 \%)\end{array}$ \\
\hline 1974 & Hymenolepis diminuta & 342 & $\begin{array}{l}259 \\
(75 \cdot 7 \%)\end{array}$ & $\begin{array}{l}13 \\
(3 \cdot 8 \%)\end{array}$ & $\begin{array}{l}22 \\
(6 \cdot 4 \%)\end{array}$ & $\begin{array}{l}43 \\
(12 \cdot 6 \%)\end{array}$ & $\begin{array}{l}5 \\
(1 \cdot 5 \%)\end{array}$ & \\
\hline 1474 & Taenia sp & 326 & $\begin{array}{l}299 \\
(91 \cdot 7 \%)\end{array}$ & & $\begin{array}{l}i \\
(0.3 \%)\end{array}$ & $\begin{array}{l}11 \\
(3 \cdot 4 \%)\end{array}$ & $\begin{array}{l}2 \\
(0.6 \%)\end{array}$ & $\begin{array}{l}13 \\
(4 \cdot 0 \%)\end{array}$ \\
\hline 1654 & Taenia sp & 345 & $\begin{array}{l}310 \\
(89 \cdot 8 \%)\end{array}$ & $\begin{array}{l}2 \\
(0 \cdot 6 \%)\end{array}$ & $\begin{array}{l}7 \\
(2.0 \%)\end{array}$ & $\begin{array}{l}13 \\
(3 \cdot 8 \%)\end{array}$ & $\begin{array}{l}2 \\
(0.6 \%)\end{array}$ & $\begin{array}{l}11 \\
(3 \cdot 2 \%)\end{array}$ \\
\hline 1941 & Taenia sp & 324 & $\begin{array}{l}284 \\
(87 \cdot 8 \%)\end{array}$ & & $\begin{array}{l}3 \\
(0.9 \%)\end{array}$ & $\begin{array}{l}27 \\
(8 \cdot 3 \%)\end{array}$ & $\begin{array}{l}5 \\
(1.5 \%)\end{array}$ & $\begin{array}{l}5 \\
(1.5 \%)\end{array}$ \\
\hline 1573 & Echinococcus granulosus\# & 339 & $\begin{array}{l}234 \\
(69 \cdot 1 \%)\end{array}$ & & $\begin{array}{l}33 \\
(9 \cdot 7 \%)\end{array}$ & $(4 \cdot 1 \%)$ & 57 & $(0.3 \%)$ \\
\hline 1916 & Echinococcus granulosus\# & 322 & $\begin{array}{l}301 \\
(93.5 \%)\end{array}$ & & $\begin{array}{l}3 \\
(0.9 \%)\end{array}$ & $\begin{array}{l}5 \\
(1 \cdot 6 \%)\end{array}$ & $\begin{array}{l}12 \\
(3 \cdot 7 \%)\end{array}$ & $\begin{array}{l}\mathrm{i} \\
(0.3 \%)\end{array}$ \\
\hline 2200 & Echinococcus granulosus\# & 351 & 338 & & 6 & $(1 \cdot 1 \%)$ & 3 & \\
\hline $\begin{array}{l}2406 \\
\text { Trematodes: }\end{array}$ & Echinococcus granulosus\# & 339 & $\begin{array}{l}312 \\
(92 \cdot 0 \%)\end{array}$ & & $\begin{array}{l}18 \\
(5 \cdot 3 \%)\end{array}$ & $\begin{array}{l}6 \\
(1 \cdot 8 \%)\end{array}$ & $\begin{array}{l}2 \\
(0 \cdot 6 \%)\end{array}$ & $\begin{array}{l}1 \\
(0 \cdot 3 \%)\end{array}$ \\
\hline 1433 & Fasciola hepatica & 331 & $\begin{array}{l}177^{\star \star} \\
(53 \cdot 3 \%)\end{array}$ & & & $\begin{array}{l}17 \\
(5 \cdot 1 \%)\end{array}$ & $\begin{array}{l}130 \\
(39 \cdot 3 \%)\end{array}$ & $\begin{array}{l}7 \\
(2 \cdot 1 \%)\end{array}$ \\
\hline 1973 & Fasciola hepatica & 329 & $\begin{array}{l}290 \star \star \\
(88 \cdot 2 \%)\end{array}$ & & $\begin{array}{l}4 \\
(1 \cdot 2 \%)\end{array}$ & $\begin{array}{l}22 \\
(6 \cdot 7 \%)\end{array}$ & $\begin{array}{l}7 \\
(2 \cdot 1 \%)\end{array}$ & (1.8\%) \\
\hline 1546 & Clonorchis sinensis & 340 & $\begin{array}{l}325^{\star \star} \\
(95 \cdot 6 \%)\end{array}$ & & $(0.3 \%)$ & $\begin{array}{l}9 \\
(2 \cdot 6 \%)\end{array}$ & $\begin{array}{l}3 \\
(0 \cdot 9 \%)\end{array}$ & 2 \\
\hline 1874 & Clonorchis sinensis & 344 & $\begin{array}{l}335^{\star \star} \\
(97 \cdot 4 \%)\end{array}$ & & & $\begin{array}{l}4 \\
(1 \cdot 2 \%)\end{array}$ & & $\begin{array}{l}5 \\
(1 \cdot 4 \%)\end{array}$ \\
\hline 1684 & Schistosoma mansoni & 343 & $\begin{array}{l}309 \\
(90 \cdot 1 \%)\end{array}$ & & & & $\begin{array}{l}34 \\
(9 \cdot 9 \%)\end{array}$ & \\
\hline 1845 & Schistosoma mansoni & 342 & $(89 \cdot 8 \%)$ & $\begin{array}{l}1 \\
(0 \cdot 3 \%)\end{array}$ & $\begin{array}{l}1 \\
(0.3 \%)\end{array}$ & $\begin{array}{l}4 \\
(1 \cdot 2 \%)\end{array}$ & $(6 \cdot 4 \%)$ & $\begin{array}{l}7 \\
(2 \cdot 0 \%)\end{array}$ \\
\hline 2284 & Schistosoma mansoni & 354 & $\begin{array}{l}297 \\
(83.9 \%)\end{array}$ & $(0 \cdot 3 \%)$ & $(1 \cdot 1 \%)$ & $(1 \cdot 7 \%)$ & 27 & $\begin{array}{l}19 \\
(5 \cdot 4 \%)\end{array}$ \\
\hline 1353 & Schistosoma haematobium\#\# & 325 & 232 & & & & 92 & $(0 \cdot 3 \%)$ \\
\hline 1846 & Schistosoma haematobium\#\# & 342 & $\begin{array}{l}303 \\
(88.6 \%)\end{array}$ & $\begin{array}{l}4 \\
(1 \cdot 2 \%)\end{array}$ & & & $\begin{array}{l}34 \\
(9 \cdot 9 \%)\end{array}$ & $\begin{array}{l}i \\
(0.3 \%)\end{array}$ \\
\hline 2253 & Schistosoma haematobium\#\# & 354 & $\begin{array}{l}349 \\
(98 \cdot 6 \%)\end{array}$ & & $\begin{array}{l}3 \\
(0 \cdot 8 \%)\end{array}$ & & $\begin{array}{l}2 \\
(0 \cdot 6 \%)\end{array}$ & \\
\hline 1975 & Schistosoma mekongi & 342 & $\begin{array}{l}280 \\
(81.9 \%)\end{array}$ & $\begin{array}{l}7 \\
(2 \cdot 0 \%)\end{array}$ & (0.6\%) & $\begin{array}{l}24 \\
(7 \cdot 0 \%)\end{array}$ & $\begin{array}{l}13 \\
(3 \cdot 8 \%)\end{array}$ & $\begin{array}{l}16 \\
(4 \cdot 7 \%)\end{array}$ \\
\hline $\begin{array}{l}\text { Protozoa: } \\
1351\end{array}$ & Entamoeba coli & 326 & $\begin{array}{l}229 \\
(70 \cdot 3 \%)\end{array}$ & & $\begin{array}{l}3 \\
(0.9 \%)\end{array}$ & & $\begin{array}{l}3 \\
(0.9 \%)\end{array}$ & $\begin{array}{l}91 \\
(27 \cdot 9 \%)\end{array}$ \\
\hline 1382 & Entamoeba histolytica & 337 & 277 & 6 & $\begin{array}{l}8 \\
(2 \cdot 4 \%)\end{array}$ & $\begin{array}{l}5 \\
(1 \cdot 5 \%)\end{array}$ & & $(12 \cdot 1 \%)$ \\
\hline 1915 & Entamoeba histolytica & 321 & $\begin{array}{l}272 \\
(84 \cdot 7 \%)\end{array}$ & 3 & & 8 & $\begin{array}{l}4 \\
(1 \cdot 3 \%)\end{array}$ & 34 \\
\hline 1475 & Iodamoeba butschlii & 326 & $\begin{array}{l}279 \\
(85.6 \%)\end{array}$ & & $\begin{array}{l}2 \\
(0 \cdot 6 \%)\end{array}$ & $\begin{array}{l}8 \\
(2.5 \%)\end{array}$ & $\begin{array}{l}7 \\
(2 \cdot 1 \%)\end{array}$ & $\begin{array}{l}30 \\
(9 \cdot 2 \%)\end{array}$ \\
\hline 1574 & Chilomastix mesnili & 340 & $\begin{array}{l}235 \\
(69 \cdot 1 \%)\end{array}$ & & & $(21 \cdot 5 \%)$ & $\begin{array}{l}19 \\
(5 \cdot 6 \%)\end{array}$ & $(3 \cdot 8 \%)$ \\
\hline 2321 & Chilomastix mesnili & 336 & $\begin{array}{l}242 \\
(72 \cdot 0 \%)\end{array}$ & & & $\begin{array}{l}47 \\
(14 \cdot 0 \%)\end{array}$ & $\begin{array}{l}30 \\
(8 \cdot 9 \%)\end{array}$ & $\begin{array}{l}17 \\
(5 \cdot 1 \%)\end{array}$ \\
\hline 1404 & Endolimax nana & 323 & $\begin{array}{l}189 \\
(58 \cdot 5 \%)\end{array}$ & & $\begin{array}{l}17 \\
(5 \cdot 3 \%)\end{array}$ & $\begin{array}{l}39 \\
(12 \cdot 1 \%)\end{array}$ & $\begin{array}{l}57 \\
(17 \cdot 6 \%)\end{array}$ & $\begin{array}{l}21 \\
(6 \cdot 5 \%)\end{array}$ \\
\hline 1547 & $\begin{array}{l}\text { Giardia lamblia } \\
\text { (cysts) }\end{array}$ & 340 & $\begin{array}{l}333 \\
(97.9 \%)\end{array}$ & & & $(0 \cdot 3 \%)$ & $\begin{array}{l}5 \\
(1 \cdot 5 \%)\end{array}$ & i \\
\hline 1954 & $\begin{array}{l}\text { Giardia lamblia } \\
\text { (cysts) }\end{array}$ & 330 & $\begin{array}{l}308 \\
(93 \cdot 3 \%)\end{array}$ & & & $\begin{array}{l}3 \\
(0.9 \%)\end{array}$ & $\begin{array}{l}19 \\
(5 \cdot 8 \%)\end{array}$ & \\
\hline 1972 & $\begin{array}{l}\text { Giardia lamblia } \\
\text { (cysts) }\end{array}$ & 329 & $\begin{array}{l}313 \\
(95 \cdot 2 \%)\end{array}$ & & & $\begin{array}{l}6 \\
(1 \cdot 8 \%)\end{array}$ & 6 & $\begin{array}{l}4 \\
(1 \cdot 2 \%)\end{array}$ \\
\hline 1711 & $\begin{array}{l}\text { Giardia lamblia } \\
\text { (trophozoites) }\end{array}$ & 343 & $\begin{array}{l}300 \\
(87 \cdot 5 \%)\end{array}$ & & & $\begin{array}{l}5 \\
(1.5 \%)\end{array}$ & $\begin{array}{l}38 \\
(11 \cdot 0 \%)\end{array}$ & \\
\hline $\begin{array}{l}\text { Coccidia: } \\
1434\end{array}$ & Cryptosporidium sp & 318 & $\begin{array}{l}286 \\
(89.9 \%)\end{array}$ & & & & $\begin{array}{l}32 \\
(10 \cdot 1 \%)\end{array}$ & \\
\hline 1761 & Cryptosporidium sp & 340 & $\begin{array}{l}309 \\
(90.9 \%)\end{array}$ & & & & $\begin{array}{l}31 \\
(9 \cdot 1 \%)\end{array}$ & \\
\hline 1875 & Cryptosporidium sp & 340 & $\begin{array}{l}309 \\
(90.9 \%)\end{array}$ & & & & $\begin{array}{l}31 \\
(9 \cdot 1 \%)\end{array}$ & \\
\hline 2176 & Cryptosporidium sp & 348 & $\begin{array}{l}332 \\
(95.4 \%)\end{array}$ & & & $\begin{array}{l}1 \\
(0.3 \%)\end{array}$ & $\begin{array}{l}15 \\
(4 \cdot 3 \%)\end{array}$ & \\
\hline 2362 & Cryptosporidium sp & 336 & $\begin{array}{l}321 \\
(95.5 \%)\end{array}$ & & & & $\begin{array}{l}15 \\
(4.5 \%)\end{array}$ & \\
\hline
\end{tabular}

*Some non-faecal parasites included. ${ }^{\star \star}$ Includes visually indistinguishable ova. †Most reports stated Strongyloides stercoralis (misidentification). ††Sputum sample containing filariform larvae of Strongyloides stercoralis. \#Hydatid cyst fluid samples. \#\#Terminal urine samples. NB: From specimen 2150 onwards, incorrect reporting of stages present was penalised in scoring. 
Table 4 Mixed faecal specimens distributed and participants' results

\begin{tabular}{|c|c|c|c|c|c|c|c|}
\hline $\begin{array}{l}\text { Specimen } \\
\text { numbers }\end{array}$ & Species & $\begin{array}{l}\text { Number of } \\
\text { laboratories }\end{array}$ & $\begin{array}{l}\text { Three parasites } \\
\text { found }{ }^{*}\end{array}$ & $\begin{array}{l}\text { Two parasites } \\
\text { found }\end{array}$ & $\begin{array}{l}\text { One parasite } \\
\text { found }{ }^{\star}\end{array}$ & $\begin{array}{l}\text { No parasites } \\
\text { found }\end{array}$ & $\begin{array}{l}\text { Unexpected } \\
\text { parasites only }\end{array}$ \\
\hline 1403 & \multirow{9}{*}{$\begin{array}{l}\text { Hookworm sp and } \\
\text { Trichuris trichiura } \\
\text { Endolimax nana and } \\
\text { Entamoeba coli } \\
\text { Entamoeba histolytica and } \\
\text { Entamoeba coli } \\
\text { Entamoeba histolytica and } \\
\text { Entamoeba coli } \\
\text { Chilomastix mesnili and } \\
\text { Entamoeba coli } \\
\text { Entamoeba histolytica, Entamoeba coli, } \\
\text { and Giardia lamblia } \\
\text { Hymenolepis nana, Entamoeba coli } \\
\text { and rhabditiform larvae of } \\
\text { Strongyloides stercoralis } \\
\text { Ascaris lumbricoides and } \\
\text { Hookworm sp } \\
\text { Taenia sp and Endolimax nana }\end{array}$} & 323 & N/A & \multirow{7}{*}{$\begin{array}{l}177 \\
(54 \cdot 8 \%) \\
181 \\
(53 \cdot 2 \%) \\
276 \\
(80 \cdot 3 \%) \\
97^{\star \star} \\
(28 \cdot 9 \%) \\
135 \\
(41 \cdot 8 \%) \\
201 \\
(57 \cdot 6 \%) \\
102 \\
(28 \cdot 3 \%)\end{array}$} & \multirow{7}{*}{$\begin{array}{l}135 \\
(41 \cdot 8 \%) \\
125 \\
(36 \cdot 8 \%) \\
61 \\
(17 \cdot 7 \%) \\
203 \\
(60 \cdot 4 \%) \\
183 \\
(56 \cdot 7 \%) \\
106 \\
(30 \cdot 4 \%) \\
44 \\
(12 \cdot 2 \%)\end{array}$} & \multirow{3}{*}{$\begin{array}{l}10 \\
(3 \cdot 1 \%) \\
16 \\
(4 \cdot 7 \%)\end{array}$} & \multirow{8}{*}{$\begin{array}{l}1 \\
(0.3 \%) \\
18 \\
(5 \cdot 3 \%) \\
7 \\
(2 \cdot 0 \%) \\
10 \\
(3.0 \%) \\
3 \\
(0.9 \%) \\
5 \\
(1.4 \%) \\
2 \\
(0.6 \%)\end{array}$} \\
\hline 1627 & & 340 & N/A & & & & \\
\hline 1653 & & 344 & N/A & & & & \\
\hline 2322 & & 336 & N/A & & & \multirow{5}{*}{$\begin{array}{l}26 \\
(7 \cdot 7 \%) \\
2 \\
(0 \cdot 6 \%) \\
9 \\
(2 \cdot 6 \%) \\
2 \\
(0 \cdot 6 \%)\end{array}$} & \\
\hline 1762 & & 323 & N/A & & & & \\
\hline 2150 & & 349 & $\begin{array}{l}28 \\
(8.0 \%)\end{array}$ & & & & \\
\hline 2175 & & 360 & $\begin{array}{l}210 \\
(58 \cdot 3 \%)\end{array}$ & & & & \\
\hline 2198 & & 354 & N/A & $\begin{array}{l}322 \\
(91 \cdot 0 \%)\end{array}$ & $\begin{array}{l}32 \\
(9 \cdot 0 \%)\end{array}$ & & \\
\hline 2254 & & 354 & N/A & $\begin{array}{l}155 \\
(43.8 \%)\end{array}$ & $\begin{array}{l}167 \\
(47 \cdot 2 \%)\end{array}$ & $\begin{array}{l}19 \\
(5 \cdot 3 \%)\end{array}$ & $\begin{array}{l}13 \\
(3 \cdot 7 \%)\end{array}$ \\
\hline
\end{tabular}

N/A: Not applicable.

* May include the presence of unexpected parasites or obvious misidentification of parasites present.

$\star \star$ This specimen contained very low cyst numbers.

Table 5 Distribution of cumulative scores for blood/tissue and faecal specimens

\begin{tabular}{|c|c|c|c|c|c|}
\hline Per cent of total possible score & $100-81$ & $80-61$ & $60-41$ & $40-21$ & $<20$ \\
\hline $\begin{array}{l}\text { Faecal parasitology: } \\
\text { No of achieving laboratories } \\
\text { Cumulative per cent of } \\
\text { laboratories achieving } \\
\text { Blood parasitology: } \\
\text { No of achieving laboratories } \\
\text { Cumulative per cent of } \\
\text { laboratories achieving }\end{array}$ & $\begin{array}{l}60 \\
(22 \cdot 8 \%) \\
35 \\
(29 \cdot 2 \%)\end{array}$ & $\begin{array}{l}131 \\
(49 \cdot 8 \%) \\
56 \\
(46 \cdot 6 \%)\end{array}$ & $\begin{array}{l}55 \\
(20 \cdot 9 \%) \\
19 \\
(15 \cdot 8 \%)\end{array}$ & $\begin{array}{l}13 \\
(4 \cdot 9 \%) \\
8 \\
(6 \cdot 7 \%)\end{array}$ & $\begin{array}{l}4 \\
(1 \cdot 5 \%) \\
2 \\
(1 \cdot 7 \%)\end{array}$ \\
\hline
\end{tabular}

Cumulative scores are shown for the $\mathbf{1 2 0}$ laboratories that returned reports on all seven blood/ tissue specimens (total possible score of 16) and the 263 laboratories that returned reports on tissue specimens (total possible score of 16 ) and the 263 laboratories that returned reports
all 12 faecal specimens (total possible score of 40) distributed from June 1990-June 1991.

coal culture, ${ }^{6}$ before adding to prescreened faeces containing either no parasites or additional known parasites. Echinococcus granulosus was obtained from cyst fluid and Fasciola hepatica and Cryptosporidium spp from veterinary sources. Latex beads were purchased from Coulter Electronics Ltd (Northwell Drive, Luton, Bedfordshire, England). The amastigotes of Leishmania spp were obtained from impression smears of hamster spleen and the Trypanosoma spp from the blood of infected rats. Faecal samples were homogenised until semi-liquid using an industrial blender, parasite numbers were assessed using the modified formol-ether method ${ }^{6}$ and faecal suspensions for distribution were diluted with $10 \% \mathrm{v} / \mathrm{v}$ formol-water ( 1 in 10 dilution of $37-41 \% \mathrm{w} / \mathrm{v}$ formaldehyde) as necessary to adjust the concentration of parasites. Specimens distributed as concentrates were produced from pooled concentrates of a single specimen using the modified formol-ether method. Faecal samples were emulsified in $10 \% \mathrm{v} / \mathrm{v}$ formol-water, filtered to remove coarse material; faecal fats were extracted by vortexing the filtrate in diethyl ether and then parasites were sedimented by centrifugation. Faecal concentrates and faecal suspensions were aliquoted by peristaltic dispenser into $2 \mathrm{ml}$ phials which were then sealed with a rubber stopper and an aluminium cap.

Thin blood smears were fixed in methanol for one minute and stained with Giemsa stain ( 1 in 20 dilution for one hour at $\mathrm{pH} 6.8$ or $\mathrm{pH}$
$7 \cdot 2$ depending on the parasite present). Slides of tissue parasites were distributed as unstained impression smears or as tissue fluid dried on to slides and fixed in methanol.

The quality of the samples and specimens was checked before and after bottling. For faecal samples, concentrations were made throughout the preparation process and following final dilution of the sample. Ten (2\%) phials were selected at random from about 500 phials bottled and the numbers of all parasites present in the samples were counted. Five (2\%) samples selected randomly from the 250 slides made from a blood specimen were examined for adequate staining and parasite morphology. Five samples of each faecal specimen and two samples of each blood specimen were returned by selected participants to HTD where they were examined to exclude deterioration of the specimen between preparation and receipt by the participants.

Specimens were labelled and packed in accordance with United Kingdom, and where appropriate, international regulations for postage of pathological specimens.

\section{DOCUMENTS}

Documents accompanied each specimen informing participants of the type of specimen, the examination required, any hazards associated with the specimen and clinical details leading to the request for examination. A questionnaire requesting details of methods used was distributed on one occasion. United Kingdom participants were asked to return reports of their results to the HTD within three weeks of distribution and overseas participants within four weeks. Following assessment and scoring of results at HTD, a summary of performance was sent to participants together with an individual computer printout showing details of performance both with current specimens and those examined over the previous 12 months, the mean of all participants' scores, and the standard error of the mean. Performance during the year was summarised for each laboratory in an annual report and individual printout of results. Educational text was dis- 
tributed on numerous occasions where an unusual parasite was distributed or where problems in performance were noted.

SCORING SCHEME

A score was allocated for each result returned by participants. Until late 1989 , a four point scoring scheme based on that used in most of the other microbiology quality assessment schemes was used. ${ }^{7}$ A score of 2 was awarded for fully correct results, 1 for partially correct results, -1 where unexpected pathogens were reported and 0 for unexpected non-pathogens, no parasites found, or inadequate identification of parasites. In 1990 a more discriminatory scoring scheme was introduced, based on the addition or subtraction of points for three components of participants' reports; recognition of the presence of a parasite; the level and correctness of identification and absence of unexpected parasites. With positive specimens, two points were added for each parasite present fully and correctly identified and one point for each parasite present incompletely characterised-that is, to genus level only or the wrong stage described. Zero points were given for incorrect speciation of parasites present or for each parasite present and not found or inadequately characterised. One point was subtracted where the incorrect stage of a parasite was described in addition to the correct stage and two points were subtracted where incorrect species were described in addition to correct species and for each unexpected parasite found (up to a maximum of three). With negative specimens, two points were awarded for a correct report of no parasites found and two points was subtracted for each unexpected parasite found (up to a maximum of three). The range of scores attainable for each specimen thus depended on the number of parasites included, varying between -6 to 2 for a negative specimen or one containing a single parasite, between -6 to 4 for a specimen containing two parasites and between -6 to 6 for a specimen containing three parasites.

\section{Results}

BLOOD PARASITES

Results of United Kingdom participants with blood specimens are shown in tables 1 and 2 . As an illustrative example for a 12 month period, the distribution of cumulative scores achieved by laboratories examining all seven specimens distributed from June 1990-June 1991 is shown in table 5 . The average cumulative score for the series was $69 \cdot 1 \%$. Some $29.2 \%$ of laboratories achieved $81 \%$ or greater of their total possible scores.

FAECAL PARASITES

Results of United Kingdom participants with faecal specimens are shown in table 3 (single parasites) and table 4 (mixed parasites). Specimens 1736 and 2342 contained latex beads with a mean measurement of $9.4 \mu \mathrm{m}$, distributed as measurement exercises. With specimen 1736, 113 of 273 laboratories $(41 \cdot 4 \%)$ reported measurement within the range 9.1-10.0 $\mu \mathrm{m} \mathrm{(+/-2SD} \mathrm{from} \mathrm{mean);} \mathrm{with}$ specimen 2342, 138 of 314 laboratories $(43.9 \%)$ reported measurement within the range $9 \cdot 1-10 \cdot 0 \mu \mathrm{m}(+/-2 \mathrm{SD}$ from mean). Six negative faecal specimens were distributed, with unexpected parasites reported by between $2.6 \%$ and $17.9 \%$ of participants. A wide range of unexpected parasites were reported, including most parasites found in human intestinal infections.

The distribution of cumulative scores achieved by laboratories examining all 12 specimens distributed from June 1990-June 1991 is shown in table 5 . The average cumulative score for the series was $68.3 \%$. Some $22.8 \%$ of laboratories achieved $81 \%$ or greater of their total possible scores.

\section{QUESTIONNAIRE}

A questionnaire requesting details of methods used was distributed in August 1990 to 463 participants and completed questionnaires were received from 314. Of the 291 laboratories specifying methods used for faecal concentration, 216 used formol-ether concentration, 57 used the "Evergreen" commercial kit (Evergreen Scientific, USA), five used the "Parakit" commercial kit (Meridian, Launch Diagnostics), and four used $\mathrm{ZnSO}_{4}$ flotation. Various other methods were used by nine participants.

Of the 226 participants using one or more stains in the examination of faecal preparations and smears, 206 used iodine, 35 Sargeaunt's, 12 eosin, 10 trichrome, eight Burrow's, five acridine, five iron haematoxylin, four Giemsa and two Field's. Various other methods were used by single participants.

One or more stains for the identification of Cryptosporidium sp were used by 199 participants of which 133 used modified Ziehl Neelsen, 85 auramine, 10 safranin/methylene blue and three MWE "Rapid Crypto" (Medical Wire Equipment).

Of the 135 participants using one or more stains for blood films, 105 used Giemsa, 17 Leishman, 12 May-Grünwald Giemsa, 12 Field's and 10 modified Wright's. Various other methods were used by 11 participants.

Eyepiece graticules were used for measurement with faecal specimens by 246 participants, were not used by 65 ; three did not answer this question.

\section{Discussion}

The organisation of a quality assessment scheme for parasitology is difficult, especially for faecal parasitology. Relatively few clinically important parasites can be grown in artificial culture or propagated in laboratory animals and clinical material is the main source of supply. Few centres in developed countries see a sufficient diversity of infections to provide material for a comprehensive quality assessment scheme. It is also difficult to collect adequate volumes of faecal material for each specimen, $1 \mathrm{~g}$ of faeces being required for each participant plus additional material for ade- 
quate quality control and for providing repeat specimens to participants failing with the first. Such quantities of material are easiest to collect from inpatients with the active collaboration of ward staff. Increasingly parasitic infections are treated in the outpatient department, which aggravates the problem of supply, as does the growth of the scheme, both in the numbers of participants and in associated teaching activities. For these reasons, it has been found necessary to undertake periodic visits to the tropics to collect material for use in the scheme.

Other problems are inherent in the use of biological material. It is difficult to ensure consistency of numbers of parasites between phials, particularly where multiple parasites or low numbers are included. Semi-liquification of the sample, thorough blending, and mixing during bottling reduce sample variation. Shortage of clinical material precludes the inclusion of "text book" examples of parasites on every occasion, and some degeneration during transport may further alter morphological features, although examination by the HTD of duplicate samples returned through the post controlled this possibility. Judgement is required to ensure that in spite of these factors the morphological features of parasites distributed are representative of those encountered in the often less than ideal specimens in the clinical laboratory. As well as potentially causing heterogeneity within a single specimen,variation among specimens can also complicate comparison of participants' results with parasites distributed repeatedly. To overcome this problem, where sufficient material is available and where parasites are stable on storage, a sample is divided into quantities sufficient for two or more distributions. The absence of very low numbers of parasites other than those seen in the organising laboratory is impossible to guarantee because there is a limit to the volume of sample that can be screened. However, participants' reporting of unexpected parasites in terms of numbers and variety was far greater than could be attributed to inadequate pre-distribution screening. Performance with negative specimens has shown some variation (table 1 ) in the numbers of participants reporting the presence of unexpected parasites, probably due to differences in the content of undigested elements (such as fruit fibres, cellulose, and meat fibres), leading to misidentification as ova and larvae. The reporting of unexpected parasites in positive samples containing parasites may be caused by misidentifications of artefacts or of the parasites present (table 3). Several of the specimens listed in table 3 show high numbers of participants reporting the presence of unexpected parasites-for example, specimen 1404 $(12 \cdot 1 \%)$, specimen $1574(21 \cdot 5 \%)$, specimen $1737(38.0 \%)$, specimen $1974(12.6 \%)$ and specimen $2255(11.8 \%)$. In most cases this is due to confusion of the actual species present, whether ova or cysts, with another species of similar appearance and size. Although participants were invited to submit photomicrographs in support of such findings, this was rarely done.

Scoring of participants' results is useful in assessing overall standards of performance with time, and in providing participants with a measure of their individual performance compared with their peers. This has proved more difficult than in other areas of microbiology because of the complexity of possible combinations of response in terms of numbers of actual parasites present, unexpected parasites reported, and the level of identification made. It is rare to find single parasites in clinical specimens and to reflect this, quality assurance specimens contained up to three parasites. Identification of most parasites relies mainly on correct morphological identification and speciation is necessary to confirm or exclude pathogenicity. For this reason, unlike other microbiology subschemes, speciation of nonpathogens is required to obtain a full score. The four point scoring scheme used for most other microbiology subschemes lacked adequate discrimination; the scheme adopted for parasitology allowed more logical scoring and provided a more objective measure of performance. It is, however, more confusing for participants since the maximum score attainable is not constant, varying between 2 and 6 , depending on the numbers of parasites present in the specimen. The scoring scheme at present lacks a penalty for incorrect answers which could lead to clinical catastrophe, as for example, misdiagnosis of a Plasmodium falciparum infection.

The overall standard of performance with these quality assessment specimens varied. At best, success rates of between 90-99\% of participants achieving full scores with some specimens comparing favourably with many of the other microbiology subschemes. However, there are considerable variations in success rates where parasites have been repeatedly distributed on separate occasions (tables 1 to 4). These differences may reflect variations between specimens such as the number of species of parasites present, the variable quantity of each species present, and the state of degeneration. Comparison between specimens is further complicated by changes in the scoring scheme introduced in June 1990 and a concurrent change from faecal concentrates to faecal suspensions.

A noticeable drop in performance was noted with repeated distribution of a mixture containing cysts of Entamoeba coli and Entamoeba histolytica (table 4). The initial sample (1653) was distributed as a faecal concentrate containing moderate numbers of cysts from both species and $80.3 \%$ of participants reported a fully correct result. The second sample (2322) was distributed as a faecal suspension for a concentration containing very low numbers of cysts, with only $28.9 \%$ of participants reporting a fully correct result. This much poorer performance could possibly be attributed to inadequate concentration of the sample or a failure fully to examine the entire sample.

Repeated distribution of less commonly encountered species has resulted in an encouraging trend towards improved performance. 
For example, the percentages of laboratories achieving fully correct results in successive distributions were, for Hymenolepis nana, $61 \cdot 1 \%$ and $75 \cdot 3 \%$; for Fasciola hepatica, 53.3\% and $88.2 \%$; and for Schistosoma haematobium, $71 \cdot 4 \%, 88 \cdot 6 \%$, and $98 \cdot 6 \%$ (table 3 ).

A sputum sample containing filariform larvae of Strongyloides stercoralis was distributed as specimen 2255 (table 3). Fully correct results were achieved by $39.6 \%$ of participants. The sample also contained some yeasts which were misidentified as Pneumocystis carinii by 29 of 38 $(78.3 \%)$ of laboratories reporting unexpected parasites. Extensive testing of the sputum sample prior to distribution showed no evidence of $P$ carinii.

Poor performance of detection of malarial parasites is mainly attributable to incorrect speciation, description of the wrong stages of parasites, and diagnosis of a mixed Plasmodium infection when only one species is present. Incorrect speciation occurs through lack of familiarity with species differences such as infected cell size and shape, parasite appearance within the cell, pigment characteristics, presence or absence of Maurer's clefts or Schuffner's dots and poor knowledge of the geographical distribution of species. Unexpected parasites reported in a sample containing malarial parasites are often the result of misidentification of artefacts and staining deposit or identifying exflagellating gametocytes as Trypanasoma $\mathrm{sp}$ or Borrelia $\mathrm{sp}$. The error in estimation of percentage parasitaemias of $P$ falciparum infections appears greatest when high numbers of parasites are present, with the poorest performance noted with specimen 2323 (23\% parasitaemia) (table 2). This could be due to laboratories using inappropriate methods of estimation such as counting more than one parasite per infected cell.

Microfilariae are poorly speciated by participants. Clinical details accompanying specimens aim to aid participants in their final diagnosis of the species present. In most cases a misidentification within the group of microfilariae cannot be considered an incorrect speciation as most genera of microfilariae contain only one species. Unexpected parasites reported with microfilariae samples (table 1) usually represent misidentification of the microfilariae present, rather than the reporting of unrelated unexpected parasites, such as specimen 2151 , where $27 \cdot 1 \%$ (36 of 133) of participants reported unexpected parasites and other microfilariae accounted for $88.9 \%$ $(32 / 36)$ of these reports.

Distributions of latex beads for measurement showed that as many as $20 \%$ of participants receiving faecal distributions did not possess an eyepiece graticule and were unable to perform measurements. This is a serious concern as measurement is essential for accurate diagnosis and differentiation of parasite ova and cysts. The wide range of measurements reported indicated that graticules in use by some participants may have been incorrectly calibrated. Results from the questionnaire showed a variation in methods used for faecal concentration. The method recommen- ded by the Department of Clinical Parasitology at HTD is modified formol-ether concentration. ${ }^{8}$ The use of gauze for the filtration of coarse material from faecal samples is inappropriate as parasites, especially ova, can be retained on its surface and wire mesh should be used. The samples of faecal suspension were intended for concentration, using the entire sample provided. All statistical data provided by QAL are based on the average numbers of parasites present in one phial of sample. Those participants who did not follow the instructions and divided a sample into several aliquots or treated the specimen as a pre-prepared faecal concentrate performed poorly as a result. Laboratories achieving incorrect results are encouraged to obtain repeat samples to investigate problems. Further assistance regarding technical matters is available from the Department of Clinical Parasitology, HTD. A mechanism for offering advice and help to poorly performing laboratories through the Advisory Panel for Microbiology ${ }^{2}$ was introduced in July 1991.

Since the closure of the IMLS fellowship course in 1972, there have been fewer opportunities for specialised training in parasitology. This problem has been addressed in part by short courses in blood and intestinal parasitology held at Paddington College (Polytechnic of Central London), but these courses are oversubscribed and accessible to only a small number of participants. Other short courses are also available at the London and Liverpool Schools of Tropical Medicine and a part time MSc course in clinical parasitology is now offered by the Royal Free Hospital School of Medicine together with the London School of Hygiene and Tropical Medicine. There are currently plans to introduce, as part of the quality assessment scheme, an expanded teaching programme, offering greater accessibility to teaching for all participants. It is hoped to achieve this by running seminars in blood and intestinal parasitology at regular intervals in various locations throughout the United Kingdom.

The parasitology schemes aim to offer an interesting and challenging programme of assessment and teaching, and it is hoped that laboratories' knowledge, confidence, and interest in parasitology will continue to grow.

1 Snell JJS, de Mello JV, Gardner PS. The United Kingdom national external microbiological quality assessment scheme. f Clin Pathol 1982;35:82-93.

2 Snell JJS. United Kingdom National external microbiological quality assessment scheme for Microbiology. Eur $\mathcal{f}$ Clin Microbiol 1985;4:464-7.

3 Diamond LS, Harlow DR, Cunnick CC. A new medium for the axenic cultivation of Entamoeba histolytica and other Entamoeba. Trans $R$ Soc Trop Hygiene 1978;74:431-2.

4 Harada Y, Mori O. A new method for culturing hookworm. Yonago Acta Med 1955;1:177-9.

5 Hsieh HC. A test-tube filter-paper method for the diagnosis of Ancylostoma duodenale, Necator americanus and Strongyloides stercoralis. WHO Technical Report Series 255:27-30.

6 Garcia LS, Ash LR Diagnostic parasitology clinical laboratory manual. 2nd ed. St Louis: CV Mosby, 1979:27.

7 Allen AVH, Ridley DS. Further observations on the formolether concentration technique for faecal parasites. $\mathcal{f}$ Clin ether concentration techn
Pathol 1970;23:545-6.

8 Snell JJS, de Mello J, Phua TJ. Errors in bacteriological techniques: results from United Kingdom national external quality assessment scheme for microbiology. Med Lab Sci 1986;43:344-5. 